University of Nebraska - Lincoln

DigitalCommons@University of Nebraska - Lincoln

USDA National Wildlife Research Center - Staff Publications
U.S. Department of Agriculture: Animal and Plant Health Inspection Service

2018

\title{
Detection of Viruses from Bioaerosols Using Anion Exchange Resin
}

Joshua W. Schaeffer

Colorado State University

Jeffrey C. Chandler

USDA APHIS Wildlife Services NWRC

Margaret Davidson

Colorado State University

Sheryl L. Magzamen

Colorado State University

Alma Perez-Mendez

Leprino Foods, Inc

See next page for additional authors

Follow this and additional works at: https://digitalcommons.unl.edu/icwdm_usdanwrc

Part of the Life Sciences Commons

Schaeffer, Joshua W.; Chandler, Jeffrey C.; Davidson, Margaret; Magzamen, Sheryl L.; Perez-Mendez, Alma; Reynolds, Stephen J.; Goodridge, Lawrence D.; Volckens, John; Franklin, Alan B.; Shriner, Susan A.; and Bisha, Bledar, "Detection of Viruses from Bioaerosols Using Anion Exchange Resin" (2018). USDA National Wildlife Research Center - Staff Publications. 2176.

https://digitalcommons.unl.edu/icwdm_usdanwrc/2176

This Article is brought to you for free and open access by the U.S. Department of Agriculture: Animal and Plant Health Inspection Service at DigitalCommons@University of Nebraska - Lincoln. It has been accepted for inclusion in USDA National Wildlife Research Center - Staff Publications by an authorized administrator of DigitalCommons@University of Nebraska - Lincoln. 


\section{Authors}

Joshua W. Schaeffer, Jeffrey C. Chandler, Margaret Davidson, Sheryl L. Magzamen, Alma Perez-Mendez, Stephen J. Reynolds, Lawrence D. Goodridge, John Volckens, Alan B. Franklin, Susan A. Shriner, and Bledar Bisha 


\section{Materials List for:}

\section{Detection of Viruses from Bioaerosols Using Anion Exchange Resin}

Joshua W. Schaeffer ${ }^{1}$, Jeffrey C. Chandler ${ }^{2}$, Margaret Davidson ${ }^{1,3}$, Sheryl L. Magzamen ${ }^{1}$, Alma Pérez-Méndez ${ }^{4}$, Stephen J. Reynolds ${ }^{1}$, Lawrence D. Goodridge ${ }^{5}$, John Volckens ${ }^{6}$, Alan B. Franklin ${ }^{2}$, Susan A. Shriner ${ }^{2}$, Bledar Bisha ${ }^{7}$

${ }^{1}$ High Plains Intermountain Center for Agricultural Health and Safety, Department of Environmental and Radiological Health Sciences, Colorado State University

${ }^{2}$ National Wildlife Research Center, Wildlife Services, Animal and Plant Health Inspection Service, United States Department of Agriculture

${ }^{3}$ Western Sydney University

${ }^{4}$ Leprino Foods, Inc

${ }^{5}$ Department of Food Science and Agricultural Chemistry, McGill University

${ }^{6}$ Department of Mechanical Engineering, Colorado State University

${ }^{7}$ Department of Animal Science, University of Wyoming

Correspondence to: Bledar Bisha at bbisha@uwyo.edu

URL: https://www.jove.com/video/58111

DOI: doi:10.3791/58111

\section{Materials}

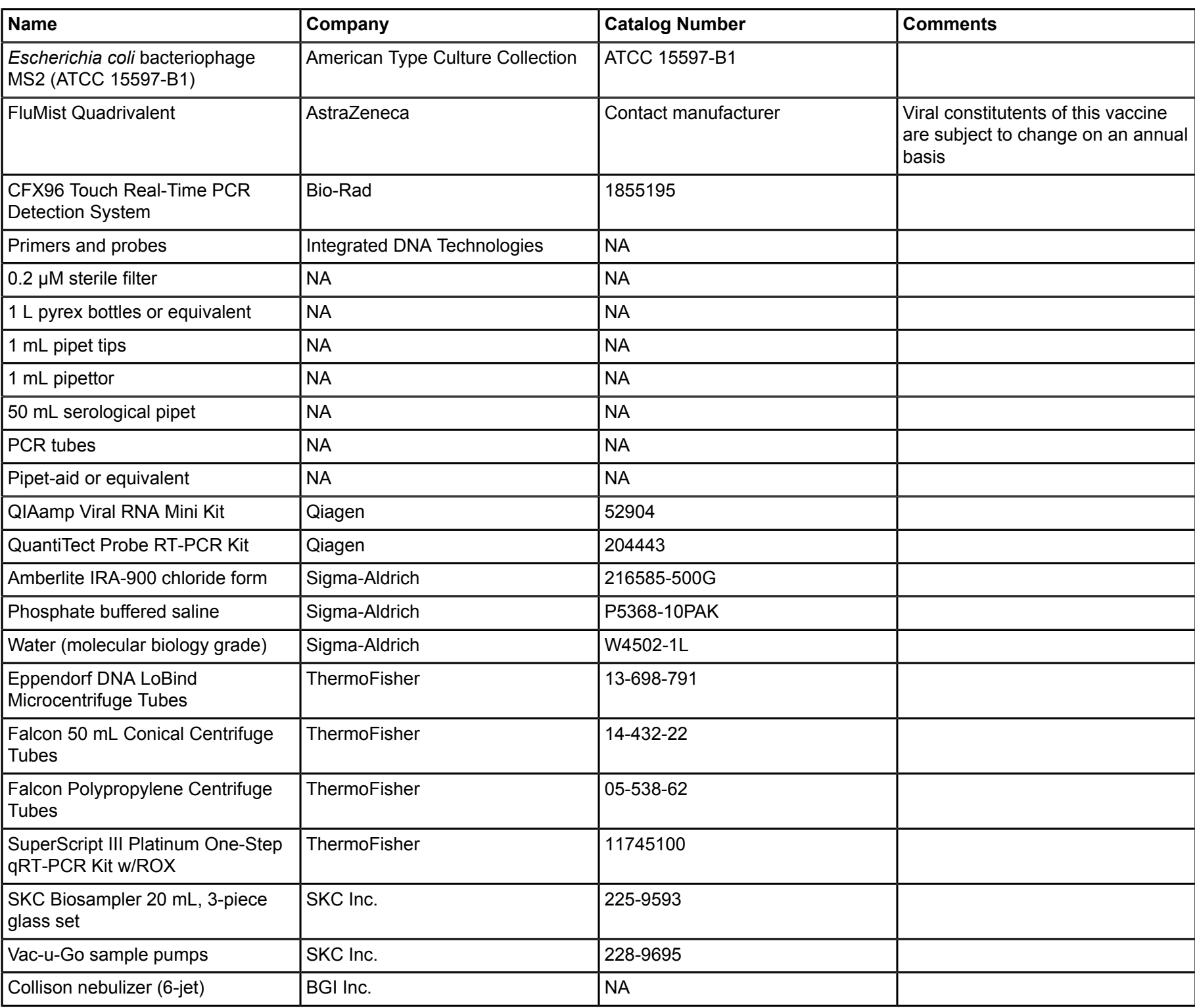




\begin{tabular}{|l|l|l|l|}
\hline HEPA capsule & PALL & 12144 & \\
\hline $\begin{array}{l}\text { Q-TRAK indoor air quality monitor } \\
8554\end{array}$ & TSI Inc. & NA & \\
\hline $\begin{array}{l}\text { Alnor velometer thermal } \\
\text { anemometer AVM440-A }\end{array}$ & TSI Inc. & NA & \\
\hline $\begin{array}{l}\text { SidePak AM510 personal aerosol } \\
\text { monitor }\end{array}$ & TSI Inc. & NA & \\
\hline Bioaerosol chamber & NA & NA & \\
\hline
\end{tabular}

